\title{
Aesthetic Surgery in Patients with Body Dysmorphic Disorder: A Grey Zone for Bioethics
}

\section{Batinic B*}

Clinic of Psychiatry, Clinical Centre of Serbia, Faculty of Philosophy, Department of Psychology, Belgrade, Serbia

*Corresponding author: Batinic B, Clinic of Psychiatry, Clinical Centre of Serbia, Pasterova 2, 11000 Belgrade, Serbia, Tel: 381637743730 ; E-mail: doubleb@eunet.rs

Rec date: March 04, 2014, Acc date: April 20, 2014, Pub date: April 28, 2014

Copyright: (C) 2014 Batinic B. This is an open-access article distributed under the terms of the Creative Commons Attribution License, which permits unrestricted use, distribution, and reproduction in any medium, provided the original author and source are credited.

\section{Short Communication}

This brief report focuses on patients diagnosed with body dysmorphic disorder who have erroneously tried to cure their problems through aesthetic surgical interventions. The highlight is on the responsibility of plastic surgeons to become more familiar with this diagnosis, as some of their clients knocked at wrong door. I would like to share my concerns about aesthetic surgery interventions in persons with body dysmorphic disorder, currently classified in Diagnostic and Statistical Manual of Mental Disorders, Fifth Edition (DSM-5) [1] in the section "Obsessive compulsive and related disorders". I will start with the case report of one of my patients (one of several cases that I have had in the last few years) who underwent, unnecessarily, two laser surgical procedures under general anesthesia before he had sought psychiatric help. The patient was a man of about 23 -years old, obsessed with redness and minor pimples on his face that caused him significant disturbance. He constantly checked his face in the mirror and demanded reassurance from family members; desperately imagining himself as perfect- he bound his happiness to perfection in his appearance, while camouflaging himself with excessive make-up. As a result, he left education, ceased sporting engagements and stopped dating and seeing friends. He was depressed and highly sensitive, with feelings of worthlessness and inadequacy. To other people, his facial complexion was normal. He fulfilled the criteria for Body dysmorphic disorder with poor insight, according to DSM-5 [1], as he was "preoccupied with perceived defects or flaws in physical appearance that are not observable or appear slight to others....that he considered as probably true.....and performed repetitive behaviors such as mirror checking, excessive grooming, reassurance seeking and camouflaging with make-up...with marked impairment in psychosocial functioning". He underwent two laser resurfacing procedures under general anesthesia in three years with only temporary satisfaction. Finally, he achieved body dysmorphic symptoms remission after a year of psychiatric treatment which included pharmacotherapy and psychotherapy. In such cases, it is obvious that it is not possible to relieve psychological problems of obsession with body image through aesthetic surgical interventions, especially in patients with poor or absent insight. Eisen et al. [2] found that $39 \%$ of patients with body dysmorphic disorder are delusional and do not recognize that their beliefs are due to a mental illness. In the described case, aesthetic surgery could be just simply a compulsive act that brings only temporary anxiety relief, but which in the long-term maintains and worsens the disorder [3]. Surgery has to be done repeatedly, because there is no "perfect solution", as it is not about a real physical problem, but due to an obsession with body image. The question that arises is whether the surgery intervention is in the "patient's best interest" and in accordance with basic ethical principles of doing good. We should be especially concerned as reported lifetime rates of suicidal ideation in patients with body dysmorphic disorder is approximately $80 . \%$ and lifetime rates of suicide attempts is $24-28 \%$ $[4,5]$. So, could we avoid mistakes through obligatory psychological assessment tests that every plastic surgery client would have to undergo in order to establish whether the desire for surgery is part of a psychiatric disorder, which helps in patient selection for aesthetic surgery intervention or psychiatric treatment. According to DSM-5 [1], the current prevalence of body dysmorphic disorder among plastic surgery patients is $7-8 \%$. Plastic surgeons should not ignore the existence of psychological problems of certain of their patients. As in psychiatry, where psychiatrists should seek to exclude any medical condition, why would plastic surgeons not be expected to specifically question their patients about symptoms of body dysmorphic disorder, to first exclude psychological disorder. Until this issue of bioethics is addressed, we will have more patients with body dysmorphic disorder in plastic surgery clinics than in psychiatric care.

\section{References}

1. American Psychiatric Association, (2013) Diagnostic and Statistical Manual of Mental Disorders, Fifth Edition, Arlington, VA, American Psychiatric Association.

2. Eisen JL, Phillips KA, Coles ME, Rasmussen SA (2004) Insight in obsessive compulsive disorder and body dysmorphic disorder. Compr Psychiatry 45: 10-15.

3. Phillips KA, Grant J, Siniscalchi J, Albertini RS (2001) Surgical and nonpsychiatric medical treatment of patients with body dysmorphic disorder. Psychosomatics 42: 504-510.

4. Phillips KA, Coles ME, Menard W, Yen S, Fay C, et al. (2005) Suicidal ideation and suicide attempts in body dysmorphic disorder. J Clin Psychiatry 66: 717-725.

5. Phillips KA (2007) Suicidality in Body Dysmorphic Disorder. Prim psychiatry 14: 58-66. 